\section{(2) OPEN ACCESS}

\title{
Single-cell RNA-seq analysis identifies meniscus progenitors and reveals the progression of meniscus degeneration
}

\author{
Hao Sun (10 , ${ }^{1,2}$ Xingzhao Wen, ${ }^{2}$ Hongyi Li, ${ }^{2}$ Peihui Wu, ${ }^{2}$ Minghui Gu, ${ }^{2}$ Xiaoyi Zhao, ${ }^{2}$ \\ Ziji Zhang, ${ }^{2}$ Shu Hu, ${ }^{2}$ Guping Mao, ${ }^{2}$ Ruofan Ma, ${ }^{1}$ Weiming Liao, ${ }^{2}$ Zhiqi Zhang (i) ${ }^{2}$
}

\begin{abstract}
Handling editor Josef $S$
Smolen

- Additional material is published online only. To view please visit the journal online (http://dx.doi.org/10.1136/ annrheumdis-2019-215926).

'Department of Orthopedics, Sun Yat-Sen Memorial Hospital, Guangzhou, China

${ }^{2}$ Department of Joint Surgery, Sun Yat-sen University First Affiliated Hospital, Guangzhou, China
\end{abstract}

\section{Correspondence to}

Dr Zhiqi Zhang, Department of Joint Surgery, Sun Yat-sen

University First Affiliated Hospital, Guangzhou 510080

China; zhzhiqi@mail.sysu.edu.cn DrWeiming Liao;

liaowmsysu@163.com

HS and XW contributed equally.

Received 28 June 2019

Revised 21 November 2019

Accepted 7 December 2019

Published Online First

23 December 2019

\section{ABSTRACT}

Objectives The heterogeneity of meniscus cells and the mechanism of meniscus degeneration is not well understood. Here, single-cell RNA sequencing (scRNA-seq) was used to identify various meniscus cell subsets and investigate the mechanism of meniscus degeneration.

Methods scRNA-seq was used to identify cell subsets and their gene signatures in healthy human and degenerated meniscus cells to determine their differentiation relationships and characterise the diversity within specific cell types. Colony-forming, multidifferentiation assays and a mice meniscus injury model were used to identify meniscus progenitor cells. We investigated the role of degenerated meniscus progenitor (DegP) cell clusters during meniscus degeneration using computational analysis and experimental verification.

Results We identified seven clusters in healthy human meniscus, including five empirically defined populations and two novel populations. Pseudotime analysis showed endothelial cells and fibrochondrocyte progenitors (FCP) existed at the pseudospace trajectory start. Melanoma cell adhesion molecule ((MCAM)/CD146) was highly expressed in two clusters. CD146+ meniscus cells differentiated into osteoblasts and adipocytes and formed colonies. We identified changes in the proportions of degenerated meniscus cell clusters and found a cluster specific to degenerative meniscus with progenitor cell characteristics. The reconstruction of four progenitor cell clusters indicated that FCP differentiation into DegP was an aberrant process. Interleukin $1 \beta$ stimulation in healthy human meniscus cells increased CD318+ cells, while TGF 31 attenuated the increase in CD318+ cells in degenerated meniscus cells.

Conclusions The identification of meniscus progenitor cells provided new insights into cell-based meniscus tissue engineering, demonstrating a novel mechanism of meniscus degeneration, which contributes to the development of a novel therapeutic strategy.

\section{INTRODUCTION}

Check for updates

(C) Author(s) (or their employer(s)) 2020. Re-use permitted under CC BY. Published by BMJ.

To cite: Sun $\mathrm{H}$, Wen $\mathrm{X}$ Li $\mathrm{H}$, et al. Ann Rheum Dis 2020;79:408-417
The menisci of mammals are crescent-shaped tissues, comprised a medial and a lateral component. ${ }^{1}$ The meniscus plays an important role in joint stability, shock absorption, distribution of contact forces, joint lubrication and proprioception. The vascularisation of the meniscus decreased with ageing. The meniscus is fully vascularised during prenatal development and shortly after birth, however, only $10 \%-25 \%$ of mature meniscus contains

\section{Key messages}

What is already known about this subject?

- The cell types of meniscus contain chondrocytelike morphology cells and fibroblast-like cells. However, the variety of cell types and corresponding biological markers, as well as the biological targets for the treatment for meniscus degeneration remain elusive.

What does this study add?

- This study provides comprehensive census of human meniscus cells using single-cell RNA sequencing, and demonstrating CD146+ meniscus cells are stem/progenitor cells.

- Interleukin $1 \beta$ induced activation of degenerated meniscus progenitor cells (DegP) is a potential mechanism contributing to meniscus degeneration.

\section{How might this impact on clinical practice or} future developments?

- CD146+ meniscus cells have potential in meniscus tissue engineering, and DegP could be a possible therapeutic target for meniscus degeneration.

blood vessels. ${ }^{2}$ According to these differences in blood supply, the meniscus can be distinguished by the outer vascular region (red zone), inner avascular region (white zone) and the red-white zone between the red and white zones. The outer zone of the meniscus contains $90 \%$ type I collagen while the inner zone contains 60\% type II collagen and 40\% type I collagen. ${ }^{3}$

The cell types of the meniscus are heterogeneous, wherein the inner region contains chondrocyte-like morphology cells and the outer region fibroblastlike cells. ${ }^{4}$ Recently, stem/progenitor cells were suggested to be present in the meniscus to promote meniscus injury repair. ${ }^{5} 6$ Gamer et al isolated meniscus stem/progenitor cells by meniscus explant culture in vitro, characterising these cells with clonogenicity properties and abundantly expressed CD44 and Sca-1.7 However, the cell-type composition and cell distribution in the menisci, as well as biochemical markers for meniscus stem cell/ progenitor for use in tissue engineering, remain to be elucidated. 
The relationship between meniscus degeneration and knee osteoarthritis (OA) is complex. Meniscus degenerative tears were found to be associated with increased cartilage loss in the same compartment, especially in posterior horn tears. ${ }^{89}$ Fuller et al found that both inner and outer zone meniscal cells are responsive to the inflammatory cytokines IL- $1 \alpha$ and TNF- $\alpha$ in an ovine in vitro model, which leads to cytokine-induced collagenolysis and aggrecanolysis. ${ }^{11}$ These studies demonstrated the importance of meniscus degeneration in OA development and its contribution to joint disease in general. Degenerative meniscus accompanied by water content increased the wet weight, while collagen and total glycosaminoglycans (GAG) decreased. ${ }^{12}$ However, the variety of cell types and corresponding biological markers, as well as the biological targets for the treatment for meniscus degeneration, have not yet been fully determined.

Single-cell RNA sequencing (scRNA-seq) is a well-established and powerful method to investigate transcriptomic cell-to-cell variation, which can be used to identify various cell types and provide insights into physiological and pathological processes. ${ }^{13} 14$ Here, we used scRNA-seq to chart a comprehensive census of meniscus cells. We identified various cell subsets and their gene signatures to determine their differentiation relationships and characterise diversity within specific cell types. We also demonstrated the existence of meniscus stem/progenitor cells and their corresponding marker genes. Finally, we investigated the integral influence of meniscus degeneration on meniscus cellular heterogeneity and identified a potential therapeutic target.

\section{MATERIALS AND METHODS \\ Isolation of human meniscus cells}

Human meniscus tissues were dissected away from the synovium, and then cut into small pieces. Next, these small pieces were digested by $4 \mathrm{mg} / \mathrm{mL}$ protease (Roche 11459643001) for 1 hour and $2 \mathrm{mg} / \mathrm{mL}$ collagenase P (Roche 11213873001) for 6-10 hours.

\section{RESULTS \\ scRNA-seq census of healthy human meniscus identified seven distinct cell populations}

Our results showed that the cell quality was satisfactory for our single cell sequencing (online supplementary figure S1). To determine the cellular composition of human meniscus cells, we profiled meniscus cells from healthy human meniscus $(n=3)$ using scRNA-seq. Unbiased clustering of the meniscus resulted in seven clusters originating from healthy human meniscus, including five empirically defined populations and two novel populations (figure 1A). Concretely, the following cells were identified: (1) endothelial cells (EC, expressing CD93 and CDH5) ${ }^{15}$ (2) cartilage progenitor cells (CPC, expressing CDK1 and BIRC5), ${ }^{16}$ (3) regulatory chondrocytes (RegC, expressing BMP2 and FOSL1), ${ }^{17}$ (4) fibrochondrocytes (FC, expressing COL1A1, COL3A1 and COL6A1), ${ }^{16}{ }^{18}$ (5) prehypertrophic chondrocytes (PreHTC, expressing MMP1 and TNFAIP6), ${ }^{19} 20$ (6) fibrochondrocyte progenitors (FCP, expressing both the fibrochondrocyte genes COL1A1 and COL3A1 and the mesenchymal stem cell marker genes MCAM and MYLK) ${ }^{21}$ and (7) proliferate fibrochondrocytes (ProFC, expressing both the fibrochondrocyte gene COL1A1 and growth factors FGF7 and CTGF) ${ }^{22}$ (figure 1B,C). FC and RegC were abundant, while FCP and EC were relatively rare.

To study the distribution of different cell clusters, we used immunohistochemistry to detect marker gene expression. MYLK, a marker gene of FCP, was mainly expressed on the meniscus surface, while the RegC gene marker BMP2 was mainly expressed in the middle of the meniscus. CD93 is the marker gene of EC and is mainly expressed around the vessels in the red zone, while the PreHTC marker ZIP8 was mainly expressed in white zone. No difference was found between the red and white areas regarding the expression of ProFC marker COL1A1, FC marker COL3A1 and CPC marker CDK1 (figure 1D).

\section{Identification of population of human meniscus progenitor cells}

To investigate the relationship between the different cell clusters, we used the Monocle method to reconstruct the pseudospace trajectory. We found that EC and FCP existed at the start of the pseudospace trajectory, and ProFC located in front of FC, while PreHTC was behind of FC. FC and CPC were distributed along the trajectory, and $\mathrm{RegC}$ was mainly distributed at the end (online supplementary figure S2A,B).

Since FCP existed at the start of the pseudospace trajectory, we investigated whether it had properties characteristic of a progenitor. Pathway analysis showed that pathways involved in focal adhesion, extracellular matrix (ECM)-receptor interaction and TGF $\beta$ signalling were activated (figure $2 \mathrm{~A}$ ). FCP expressed the mesenchymal stem cell marker MCAM (CD146) (figure 2B), as well as classical markers of myofibroblasts, including ACTA2, MYLK and MYL9 (online supplementary figure S2C).

We isolated the CD146+ primary human meniscus cells using fluorescence-activated cell sorting (FACS) and found that the proportion of CD146 + meniscus cells was near 2.7\% (figure 2C). CD146 + cells had the ability to differentiate into various cell lineages, including osteoblasts and adipocytes (figure 2D). Next, we examined the clonogenicity of CD146+ cells. A total of 2000 CD146 + and CD146- cells were seeded in 12-well plates and cultured for 7 days. After culturing, the number of colonies in the CD146+ group was significantly higher than that of the colonies in the CD146- cells group (figure 2E). The fact that the cells in the CD146- group were able to form colonies suggests that another cell cluster may also have progenitor properties. CD93 is the specific marker of ECs, so we used FACS to obtain CD146+/CD93+ meniscus cells (EC) and CD146+/CD93meniscus cells (FCP). Further experiments showed that these two clusters have progenitor properties (online supplementary figure S2D).

\section{Single-cell trajectory branch points correspond to FCP differentiation}

To study the differentiation of FCP into subset clusters and the corresponding gene expression, we selected FCP, ProFC, FC, PreHTC and RegC to construct a new trajectory containing two termini corresponding to two distinct cell fates (figure 3A). The root of the trajectory was mainly populated by FCP and ProFC, while the two termini of the tree were populated by FC and PreHTC for fate 1, RegC and PreHTC for fate 2 (figure 3B). Next, we assessed the expression of genes regulated during FCP differentiation in cells at fates 1 and 2 of the trajectory. The expression of MYLK, CNN1, FGF7 and COL1A1 were found to be similar. While MYLK and CNN1 expression was markedly reduced from the root through to both fates, FGF7 and COL1A1 expression was upregulated early in FCP differentiation and downregulated in cells differentiating into both fates (figure 3B,C). ADAMTS4 and MMP1 were slightly upregulated at early stage differentiation, and notably upregulated in cells at fate 1 and downregulated at fate 2. On the contrary, FOSL1 and 
A

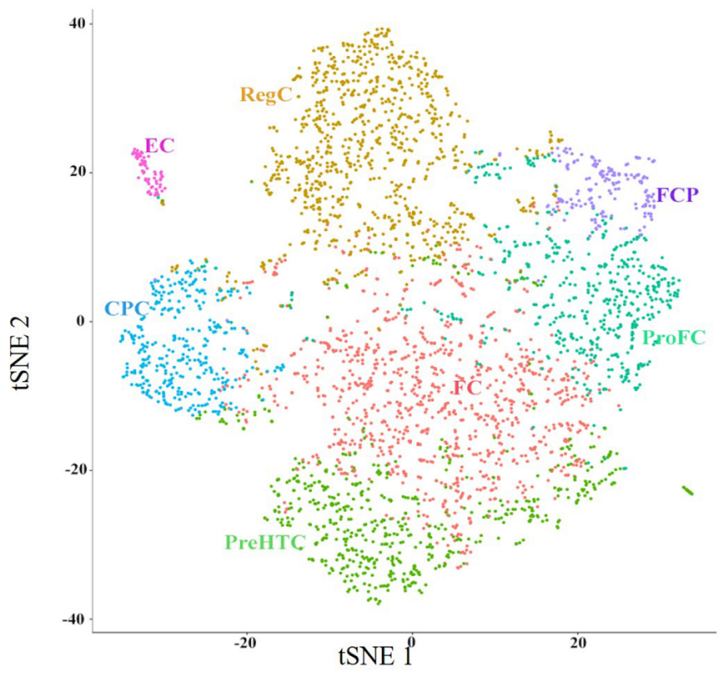

B

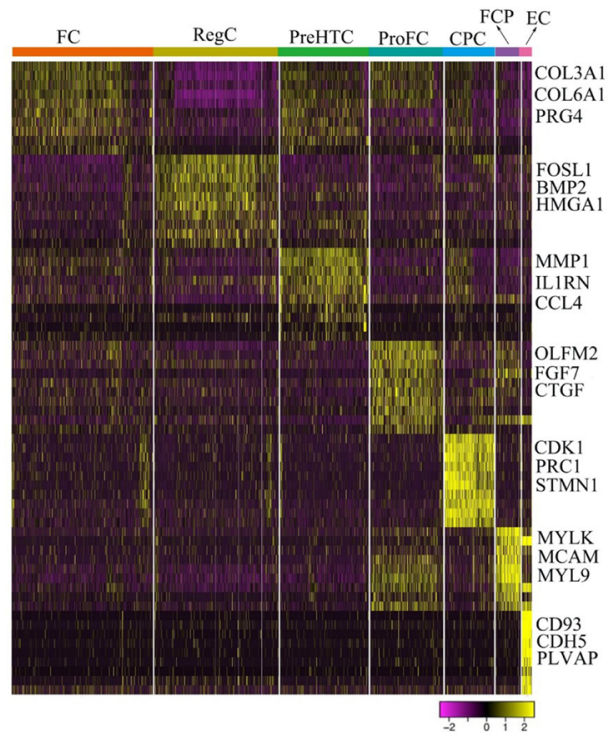

C
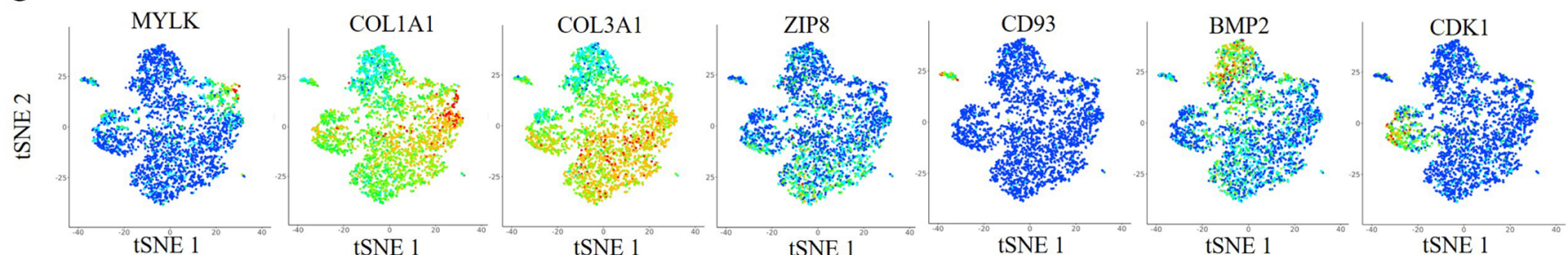

$\mathrm{D}$
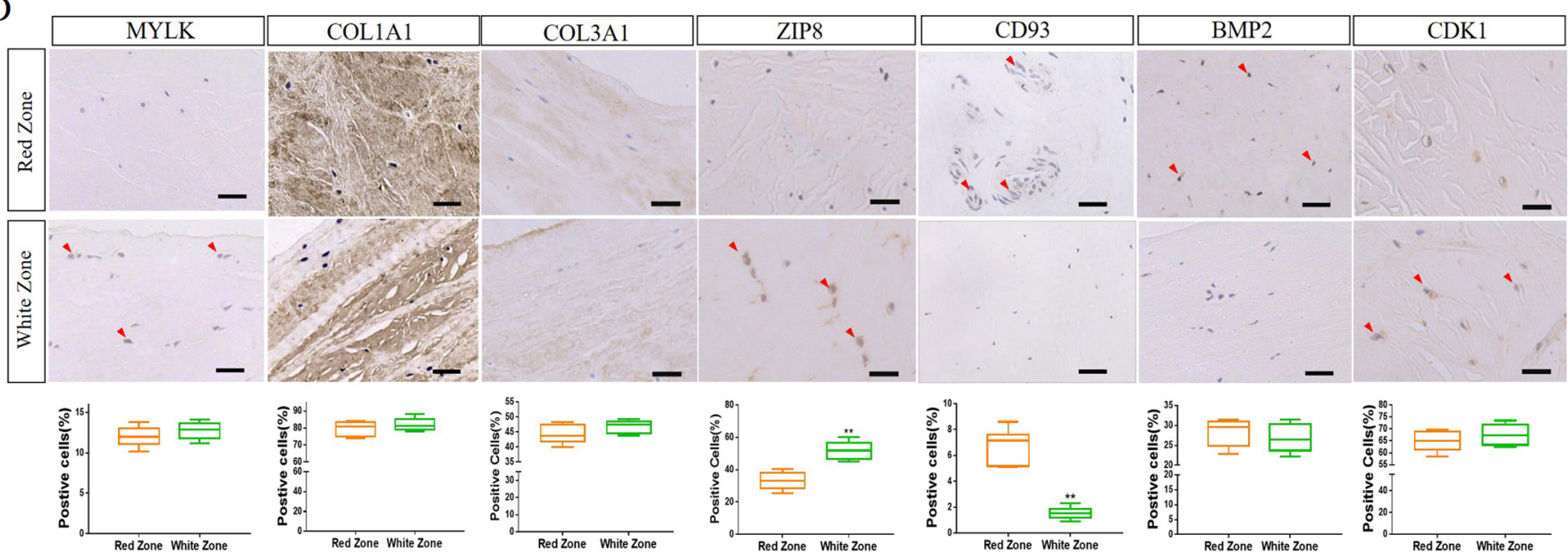

Figure 1 A single-cell atlas of healthy human meniscus. (A) Seven healthy human meniscus cell clusters. t-Distributed stochastic neighbour embedding (t-SNE) of 3639 cells (mixed with cell fractions, $n=3$ ), annotated post-hoc and coloured by clustering. (B) Heatmap revealing the scaled expression of differentially expressed genes for each cluster. (C) Dot plots showing the expression of the indicated markers for each cell cluster on the t-SNE map. (D) Representative immunohistochemistry staining of MYLK, COL1A1, COL3A1, ZIP8, CD93, BMP2 and CDK1 in white and red zones of healthy human meniscus tissues, and quantification of positive cells displayed by box plot $(n=6)$. Scale bar, $50 \mu \mathrm{m}$. ${ }^{* *} \mathrm{p}<0.01$. CPC, cartilage progenitor cells; EC, endothelial cells; FC, fibrochondrocytes; FCP, fibrochondrocyte progenitors; PreHTC, prehypertrophic chondrocytes; ProFC, proliferate fibrochondrocytes; RegC, regulatory chondrocytes.

BMP2 expression slightly decreased at cells from the root to fate 1 , but markedly increased in cells differentiating via fate 2 .

To confirm the single-cell trajectory, we analysed the meniscus developmental process in vivo and studied the expression of marker genes in the mice meniscus at 1, 2, 3, 4, 8, 26 and 52 weeks. COL1A1 expression increased gradually after birth and peaked at 4 weeks, then decreased gradually with increasing age (figure 3E). MYLK expression decreased significantly with increasing age after 3 weeks (figure 3E). These expression patterns were consistent with two different fates of the trajectory, indicating that our scRNA-seq analysis correlated with the meniscus developmental process.

\section{Systemic comparison of the single cell landscape between healthy human meniscus and degenerated meniscus}

To comprehensively assess the changes in the human meniscus during degeneration, we first evaluated the histological changes in degenerated meniscus. The healthy meniscus was negative for Safranine $\mathrm{O}$ staining, while the degenerative meniscus was positive for staining (online supplementary figure S3). In addition, 


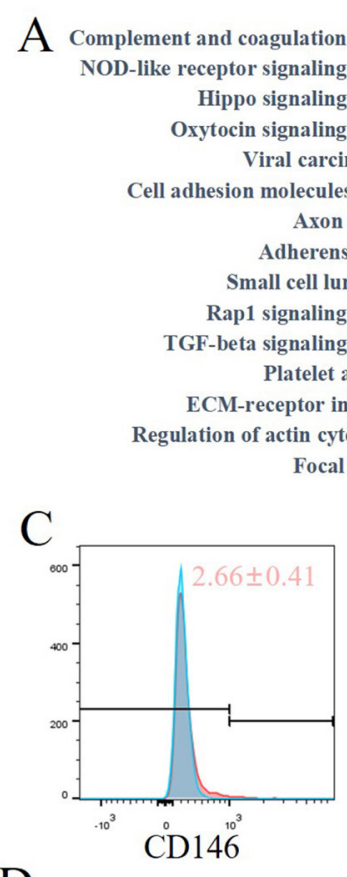

$\mathrm{D}$
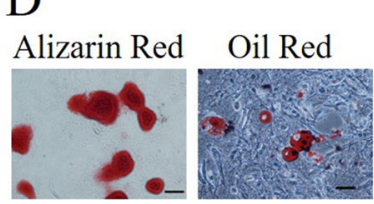

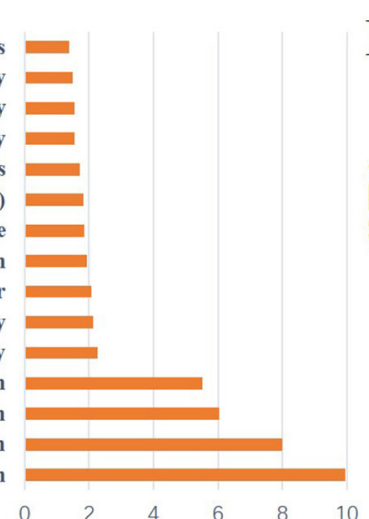

$\log 10(\mathrm{P}-$ Value $)$
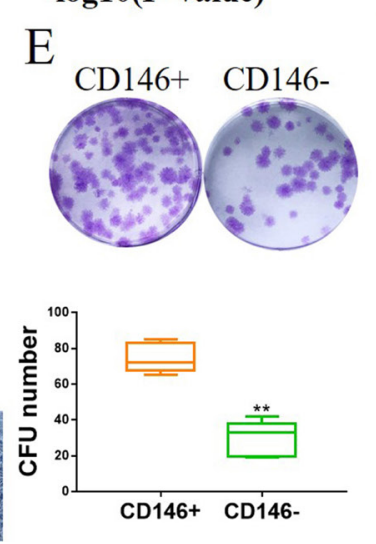

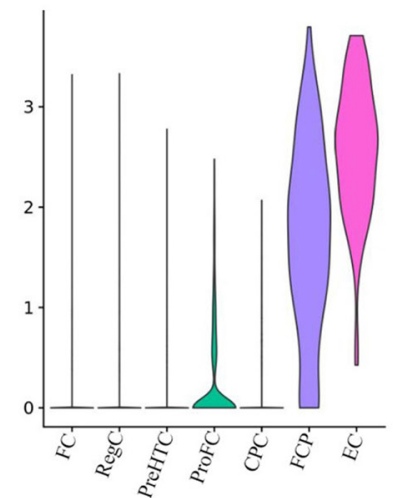

12

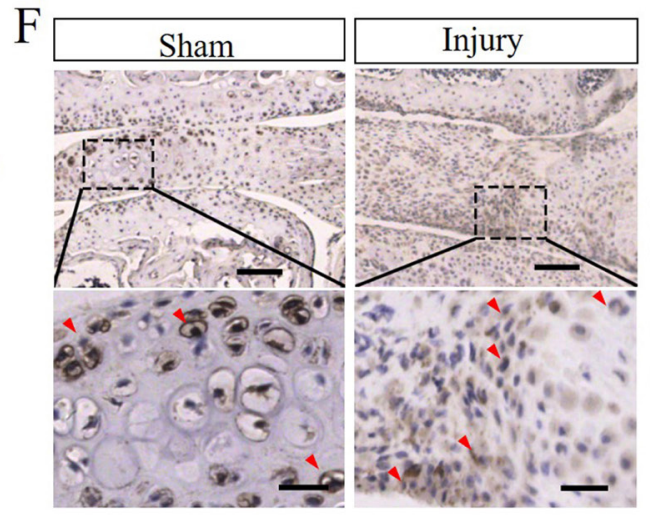

Figure 2 Identification of human meniscus progenitor cells. (A) The 15 most upregulated signal pathways in FCP. (B) Dot plots showing the MCAM expression on t-distributed stochastic neighbour embedding (t-SNE) map and Vin plot. (C) CD146 expression in healthy human meniscus cells determined by flow cytometry (mean $\pm S D ; n=3$ ). (D) Alizarin red staining and oil red staining for $C D 146+$ meniscus cells induced to osteogenic differentiation or adipogenic differentiation, respectively $(n=5)$. Scale bar, $50 \mu \mathrm{m}$. (E) Colony-forming analysis of CD146+ and CD146- healthy human meniscus cells and quantification. $n=5,{ }^{* *} p<0.01$. (F) IHC staining of MYLK in mice meniscus injury model, and quantification of positive cells. Scale bars, $200 \mu \mathrm{m}$ (top) and $50 \mu \mathrm{m}$ (bottom). $n \geq 6,{ }^{* *} p<0.01$. CFU colony forming unit; CPC, cartilage progenitor cells; $E C$, endothelial cells; FC, fibrochondrocytes; FCP, fibrochondrocyte progenitors; NOD, nucleotide-binding oligomerisation domain; PreHTC, prehypertrophic chondrocytes; ProFC, proliferate fibrochondrocytes; RegC, regulatory chondrocytes.

the collagen fibre structure on the degenerated meniscus was disorganised (Figure 4A and online supplementary figure S4). Next, we compared the scRNA-seq between healthy meniscus and degenerated meniscus (figure 4B-E). As a result, we detected significant changes in the proportions of degenerated meniscus cell clusters, including three new clusters: (1) monocyte-derived dendritic cells (MoDC, expressing CD14 and S100A9), ${ }^{23}{ }^{24}$ (2) hypertrophic chondrocytes (HTC, expressing CCL20 and EREG) $)^{25} 26$ and (3) degenerated meniscus progenitor cells (DegP), which are found in degenerated meniscus and express skeletal stem cell marker, such as GREM1 ${ }^{27}$ (figure 4C-G). Moreover, the proportion of EC and FCP expression was found to decrease in degenerated meniscus (figure 4E).

\section{Alignment of single-cell trajectories indicates DegP is a key element for meniscus degeneration}

CDCP1 (CD318) is highly expressed in DegP (online supplementary figure S4A). As such, we isolated the CD318 + primary human degenerated meniscus cells by FACS to verify the progenitor capacity. CD318+ cells were found to form colonies and differentiate into various cell lineages (online supplementary figure S4B,C), wherein DegP was a special population with progenitor characteristics, and was mainly found in the degenerative meniscus.
Next, we selected four clusters with progenitor properties, including FCP, ProFC, CPC and DegP, to construct a new trajectory. The trajectory's root was mainly populated by FCP and ProFC, while the two primary termini of the tree were populated by DegP and CPC for fate 1 , and CPC for fate 2 (figure $5 \mathrm{~A}, \mathrm{~B}$ ). Although MCAM and MYLK were highly expressed at the root of the trajectory, their expression was markedly reduced along the root through to both fates 1 and 2 (figure 5C,D). BIRC5 and CDK1 were highly expressed at the end of fate 2, while GAS1, RAB3B and CDCP1 were highly expressed at the end of fate 1 (figure 5C,D and online supplementary figure S5A). However, in normal FCP differentiation, the expression of GAS1, RAB3B and CDCP1 was markedly reduced while progressing along from the root to both fates 1 and 2 (compared with figure 3, online supplementary figure $\mathrm{S} 5 \mathrm{~B}$ ), indicating that fate 1 may be an aberrant cellular state during the degeneration process in meniscus.

We also verified the expression of marker genes by IHC staining. MYLK, an FCP marker gene, was downregulated in degenerated meniscus, while the DegP marker genes GAS1 and DNER were upregulated in degenerated meniscus, especially in areas where meniscus lesions were accompanied by cell proliferation (figure 5E).

Proinflammatory mediators, such as IL-1 $\beta$, appeared to directly influence the degradative processes in the meniscus. ${ }^{28} 29$ 
A.

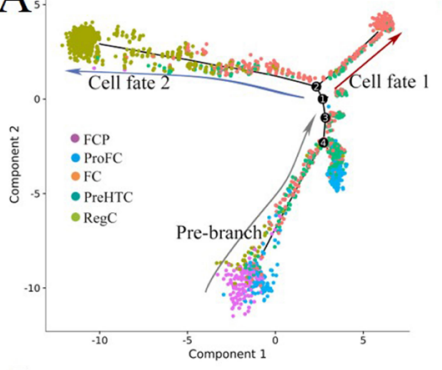

C
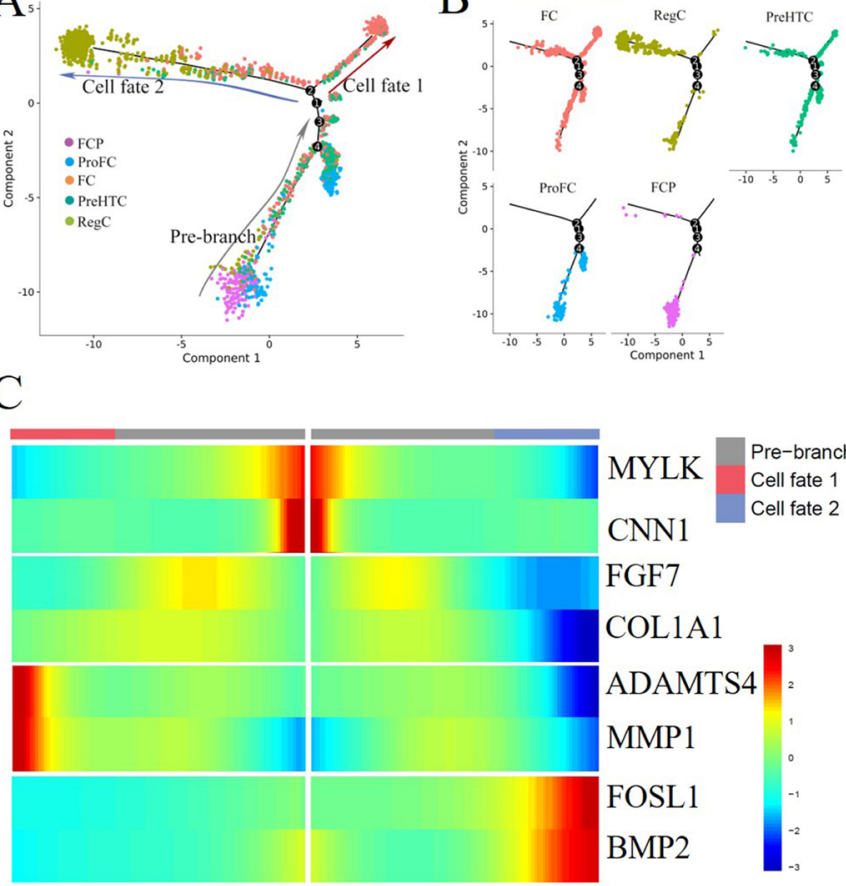

B
D

MYLK

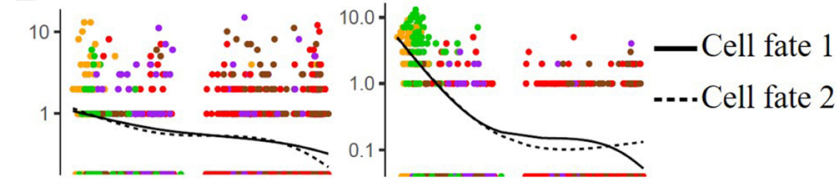

FGF7

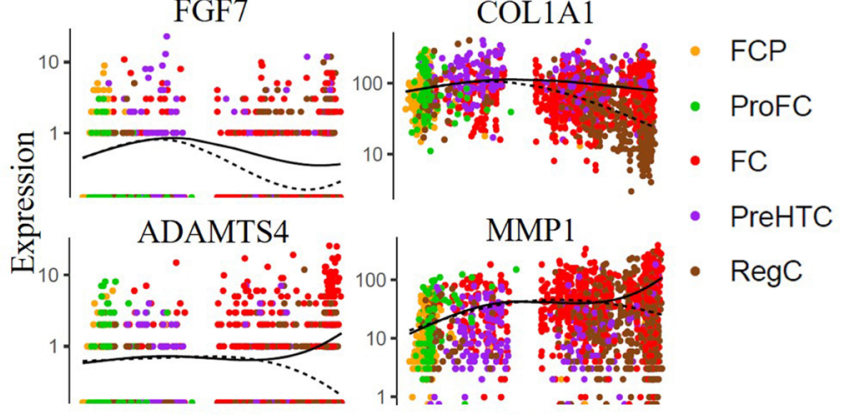

BMP2

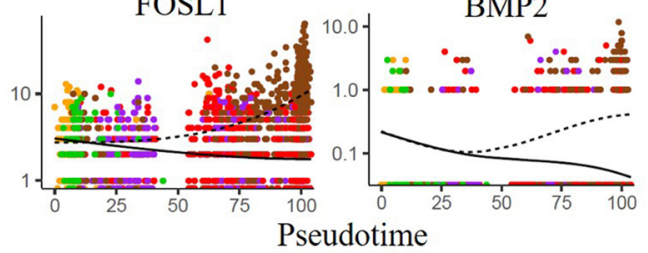

E
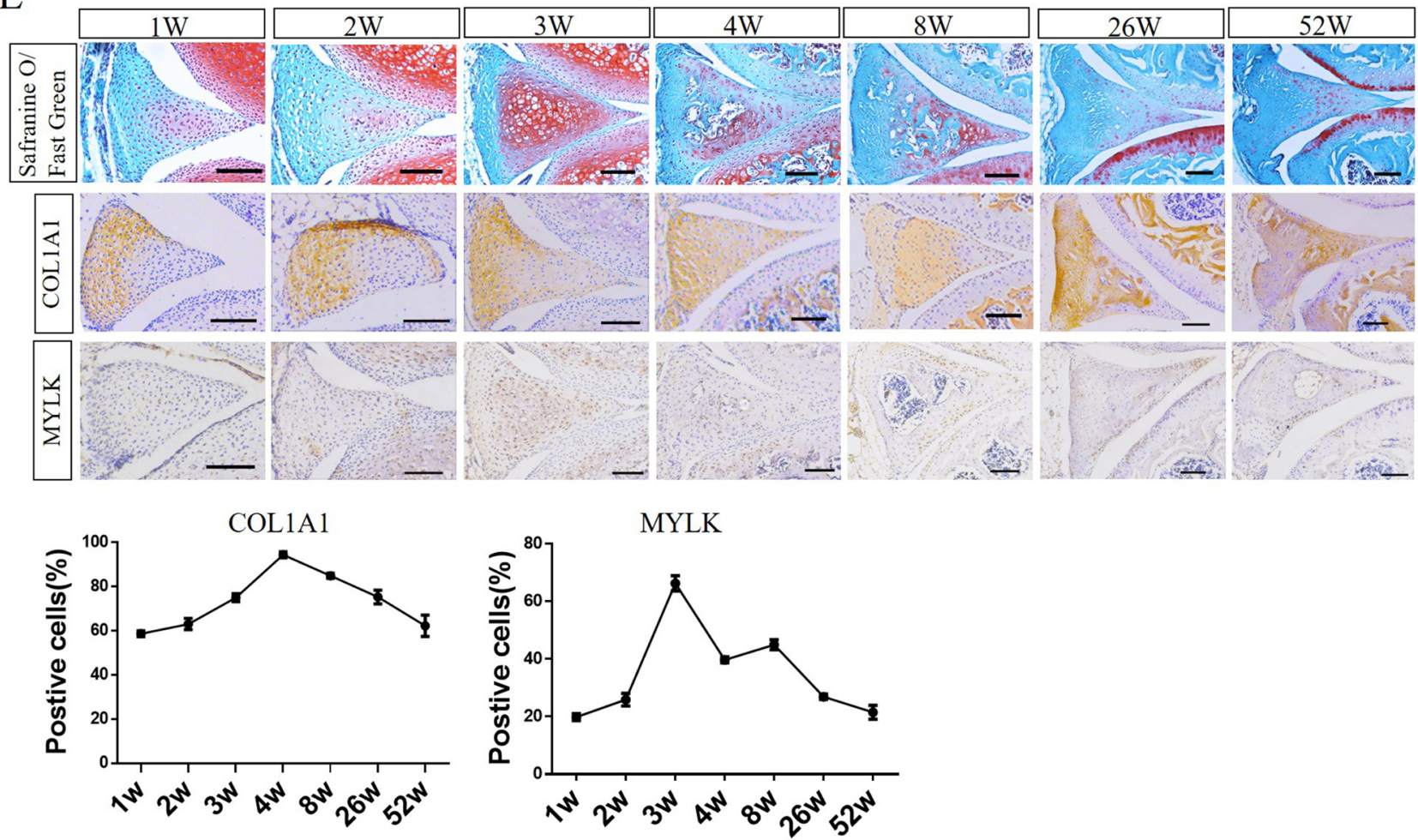

Figure 3 Single-cell trajectory branch points demonstrating FCP differentiation. (A, B) Monocle pseudotime trajectory showing the progression of FCP, ProFC, FC, PreHTC and RegC. (C) The expression of the genes in a branch-dependent manner. Each row indicates the standardised kinetic curves of a gene. The centre of the heatmap shows the kinetic curve value at the root of the trajectory. From the centre to the left of the heatmap, the kinetic curve progresses from the root along the trajectory to fate 1. Starting from the right, the curve from the root to fate 2. (D) Pseudotime kinetics of indicated genes from the root of the trajectory to fate 1 (solid line) and the cells up to fate 2 (dashed line). (E) Safranine 0/Fast Green staining and immunohistochemistry staining of COL1A1 and MYLK in mice anterior meniscus at 1, 2, 3, 4, 8, 26 and 52 weeks, and quantification of positive cells $(\mathrm{n} \geq 3)$. Scale bar, $100 \mu \mathrm{m}$. CPC, cartilage progenitor cells; EC, endothelial cells; FC, fibrochondrocytes; FCP, fibrochondrocyte progenitors; PreHTC, prehypertrophic chondrocytes; ProFC, proliferate fibrochondrocytes; RegC, regulatory chondrocytes.

Therefore, we used IL-1 $\beta(5 \mathrm{ng} / \mathrm{mL})$ to stimulate healthy human meniscus cells for 48 and 96 hours to detect any changes in CD146+ cells and CD318 + cells. IL-1 $\beta$ stimulation led to a significant reduction in CD146+ cells with an increasing stimulation time, while CD318+ cells significantly increased (figure $5 \mathrm{~F}$ ). We also used IL-1 $\beta$ to stimulate degenerated 
A

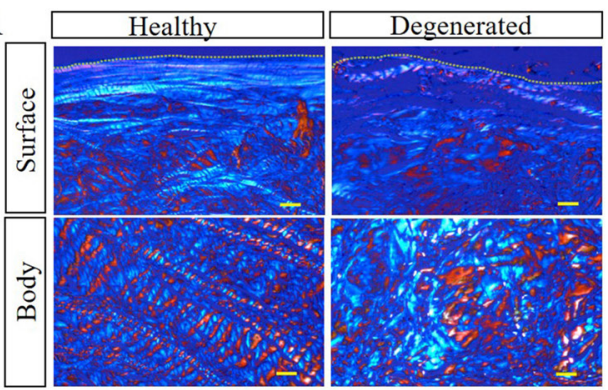

$\mathrm{D}$

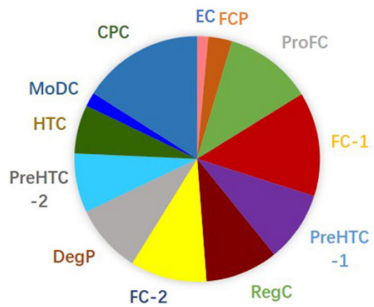

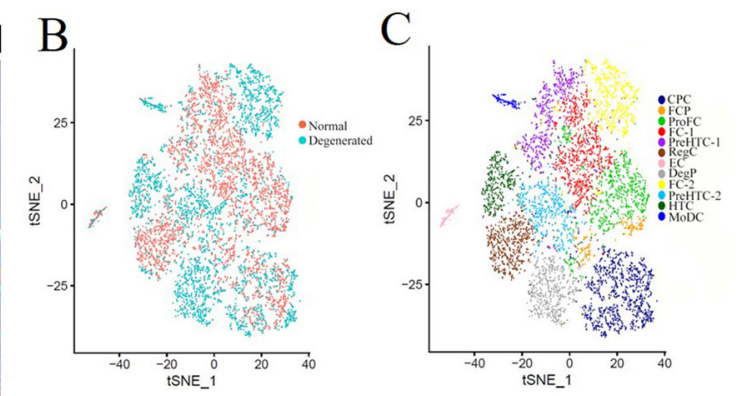

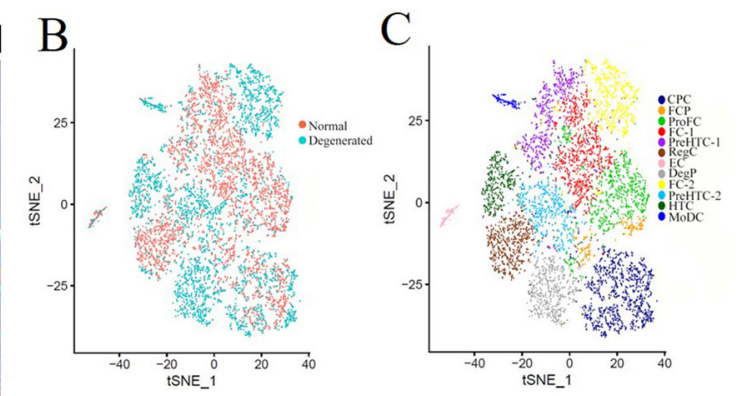

$\mathrm{E}$

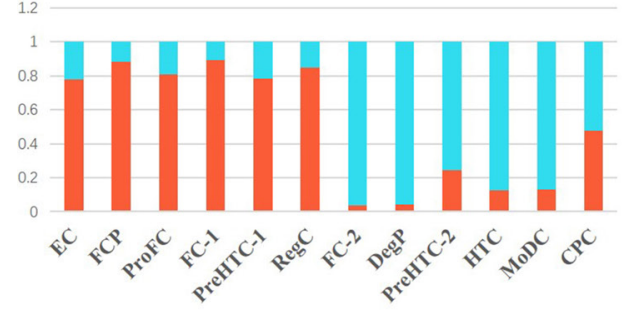

normal $\quad$ Degenerated

$\mathrm{F}$

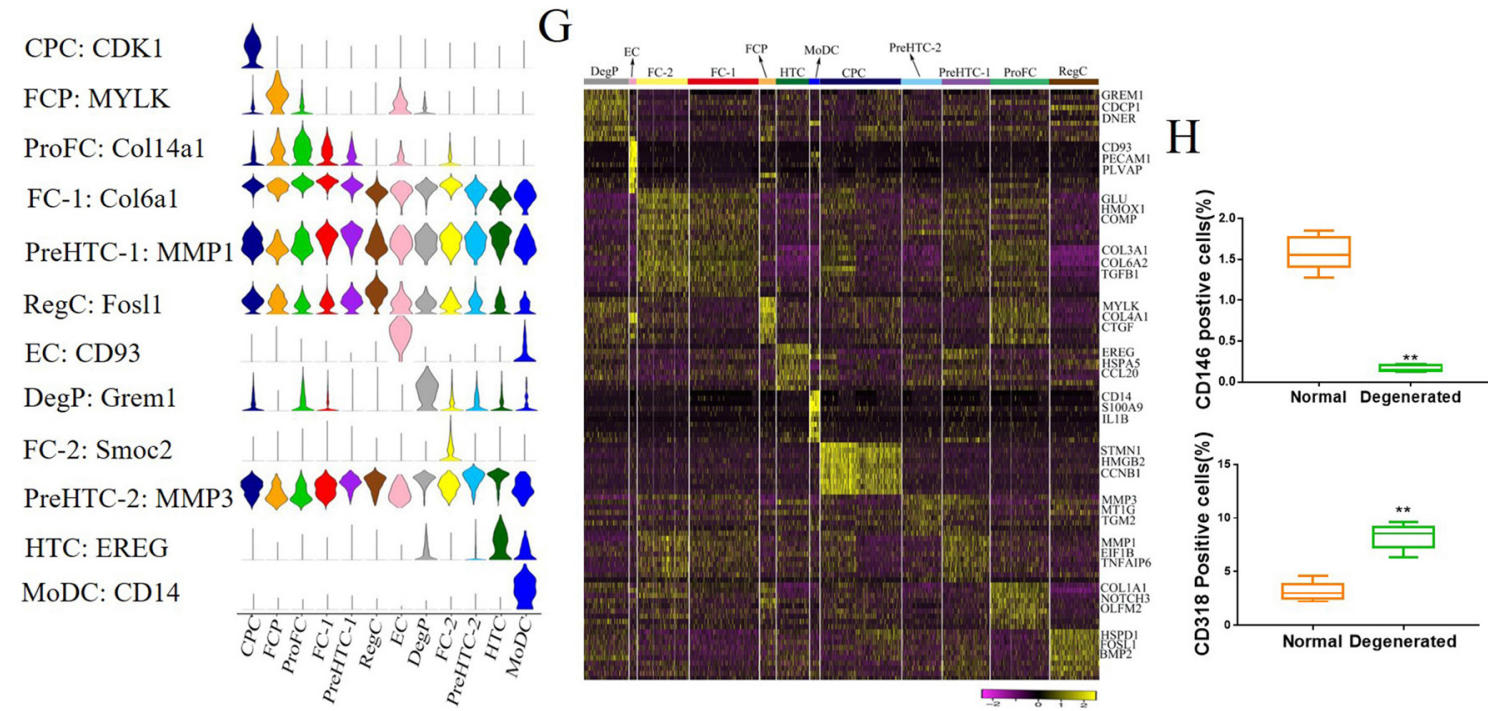

I
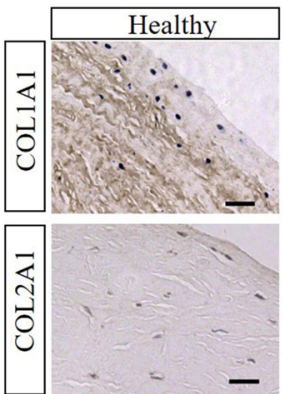
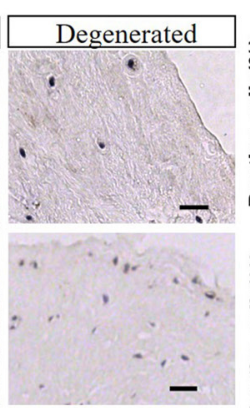

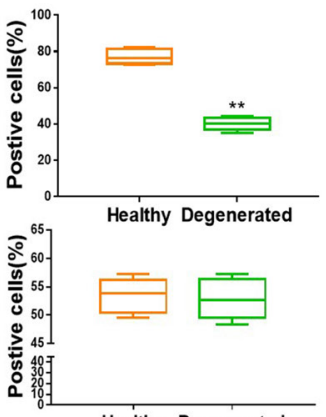

$\mathrm{J}$

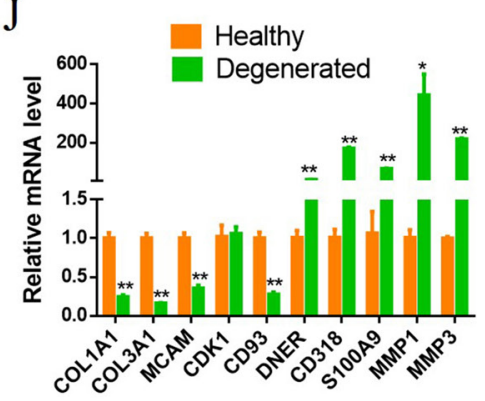

Figure 4 Comparison of the single cell landscape between healthy human meniscus and degenerated meniscus. (A) Representative polarised light microscopy images of healthy human and degenerated meniscus. The white and red colours in the angle images are $90^{\circ}$ apart in orientation. Dashed lines indicate the surface of the meniscus. Scale bar, $100 \mu \mathrm{m}$. (B) Merged t-distributed stochastic neighbour embedding (t-SNE) of single-cell RNA sequencing of healthy meniscus cells and degenerated meniscus cells. (C) Twelve healthy human and degenerated meniscus cell clusters at t-SNE. (D) Proportion of each cluster to the total cells. (E) Proportion of healthy and degenerated meniscus cells in each cluster. (F) Expression of representative marker genes in Vin plot. (G) Heatmap revealing the scaled expression of differentially expressed genes for each cluster. (H) CD146 and CD318 expression in healthy human meniscus cells and degenerated meniscus cells determined by flow cytometry. $n \geq 5,{ }^{* *} p<0.01$. (I) Representative IHC staining of COL1A1 and COL2A1 healthy human meniscus and degenerated meniscus, and quantification of positive cells. Scale bar, $50 \mu \mathrm{m}$. $\mathrm{n} \geq 5$, ${ }^{* *} p<0.01$. (J) The expression of indicated marker genes in human healthy meniscus cells and degenerated meniscus cells were detected by qRT-PCR. ${ }^{*} p<0.05,{ }^{* *} p<0.01$, otherwise, not significant. $n=3,{ }^{*} p<0.05,{ }^{* *} p<0.01$. CPC, cartilage progenitor cells; DegP, degenerated meniscus progenitor cells; EC, endothelial cells; FC, fibrochondrocytes; FCP, fibrochondrocyte progenitors; HTC, hypertrophic chondrocytes; MoDC, monocyte-derived dendritic cells; PreHTC, prehypertrophic chondrocytes; ProFC, proliferate fibrochondrocytes; RegC, regulatory chondrocytes. 

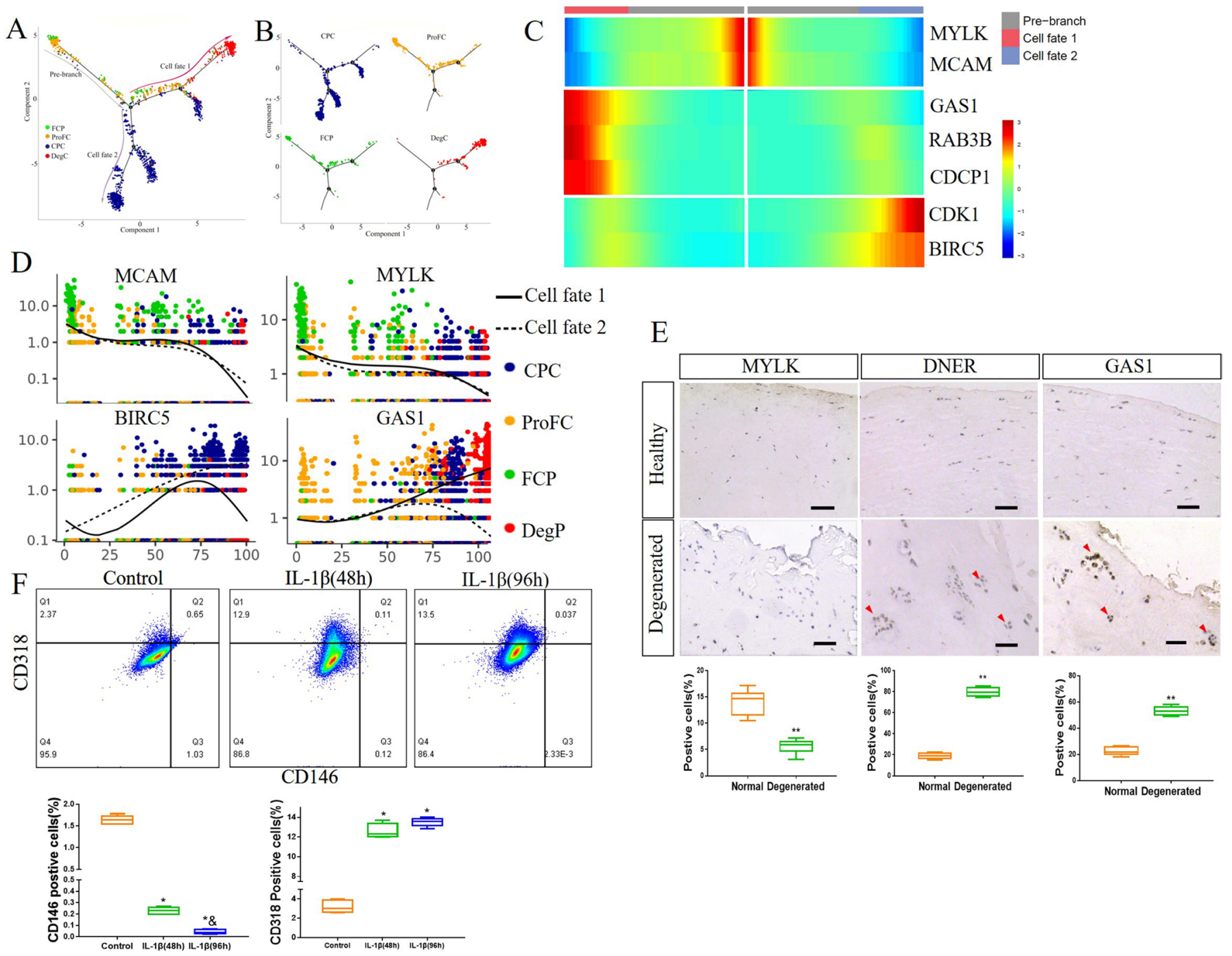

Figure 5 Identification of degenerated meniscus progenitor cells (DegP) as a key element for meniscus degeneration. (A, B) Monocle pseudotime trajectory showing the progression of FCP, ProFC, CPC and DegP. (C) From the centre to the left of the heatmap, the kinetic curve from the root along the trajectory to fate 1. Starting from the right, the curve from the root to fate 2. FCP markers MYLK and MCAM, DegP markers GAS1, Rab3B and CDCP1 and CPC markers CDK1 and BIRC5 expressed from the root to each branch. (D) Pseudotime kinetics of indicated genes from the root of the trajectory to fate 1 (solid line) and the cells up to fate 2 (dashed line). (E) Representative IHC staining of MYLK, GAS1 and DNER in healthy human meniscus and degenerated meniscus, and quantification of positive cells. Scale bar, $50 \mu \mathrm{m} . n=6,{ }^{*} p<0.01$. (F) Healthy human meniscus cells were treated with $5 \mathrm{ng} / \mathrm{mL}$ IL-1 $\beta$ for 48 hours or 96 hours. Phosphate buffer saline (PBS) was used as a negative control. CD146 and CD318 expression was determined by flow cytometry. $n \geq 5$, * versus control, $p<0.05$; \& versus IL-1 $\beta$ ( 48 hours), $p<0.05$. CPC, cartilage progenitor cells; DeP, degenerated meniscus progenitor cell; FCP, fibrochondrocyte progenitors; ProFC, proliferate fibrochondrocytes.

human meniscus cells and get similar results (online supplementary figure S5C). These results suggested that the decrease of CD146+ cells and the increase of CD318 + cells caused by various pathogenic factors such as IL-1 $\beta$, may be an important mechanism of meniscus degeneration.

\section{Activation of TGF $\beta$ signalling pathway attenuates the increase in CD318+ cells in degenerated meniscus}

Previous studies have shown that the activation of TGF $\beta$ signalling enhances the differentiation ability of meniscus progenitors. ${ }^{3031}$ Our scRNA-seq analysis and IHC staining showed that TGF $\beta 1$, a ligand of the transforming growth factor- $\beta$ (TGF $\beta$ ) signalling pathway, was highly expressed in healthy meniscus cells (figure 6A,B). We also compared the differences in gene expression between FC-1 and FC-2, PreHTC-1 and PreHTC-2, two cell types found in both healthy and degenerated meniscus.
Compared with the clusters mainly found in degenerated meniscus (FC-2 and PreHTC-2), the clusters found in healthy meniscus (FC-1 and PreHTC-1) were upregulated by the TGF $\beta$ signalling pathway (online supplementary figure S6) and highly expressed COL1A1, COL3A1 and TGF $\beta 1$ (figure 6C,D). Next, we investigated the effect of TGF $\beta 1$ on DegP. Primary human degenerated meniscus cells were treated with $5 \mathrm{ng} /$ mL TGF $\beta 1$ for 48 hours or 96 hours. Flow cytometry demonstrated that TGF $\beta 1$ treatment significantly reduced the number of CD318 + cells in a time depend manner (figure 6E), and qRT-PCR showed TGF $\beta 1$ treatment significantly increasing COL1A1, COL3A1 and CDK1 expression while decreasing CD318, S100A9, MMP1 and MMP3 expression (figure 6F), indicating that TGF $\beta 1$ may be able to delay the degeneration of meniscus. 
A
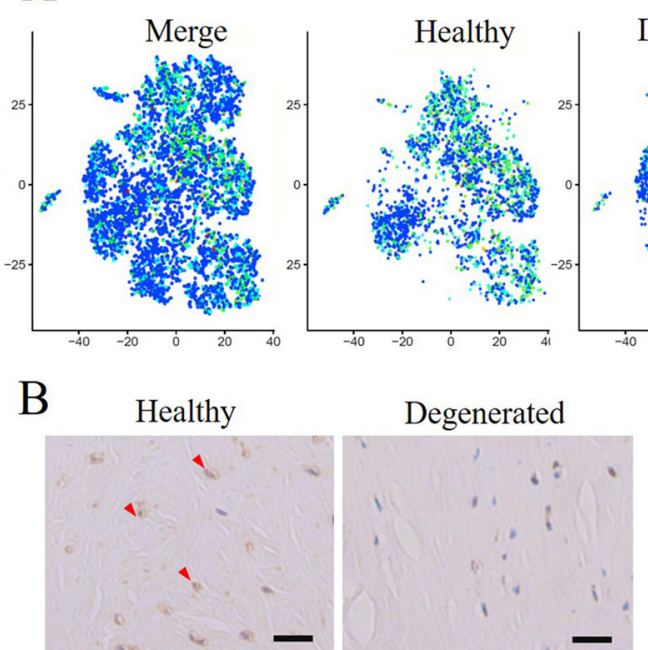

Degenerated
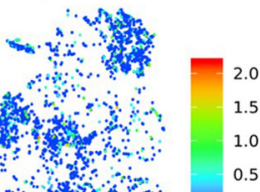

ox of

齐
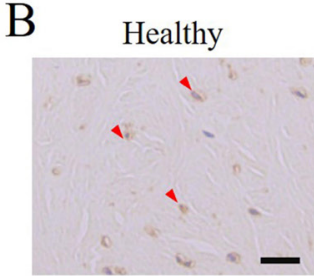

Degenerated
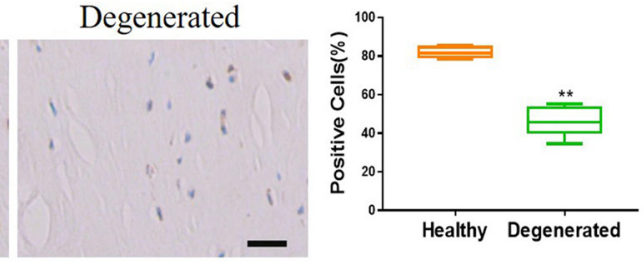

Healthy Degenerated

$\mathrm{E}$

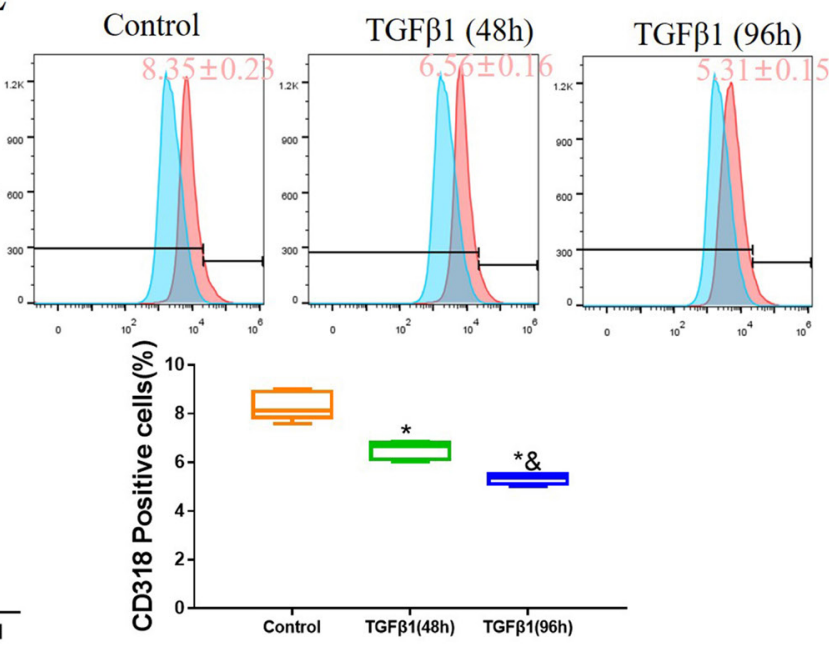

$\mathrm{F}$

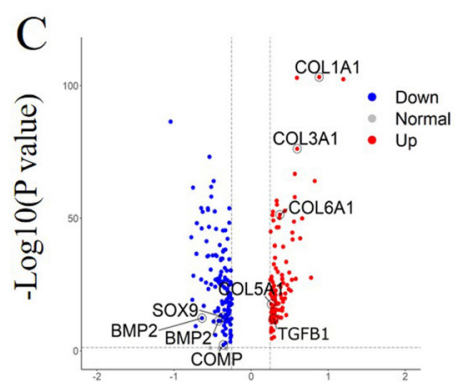

FC-1 vs FC-2

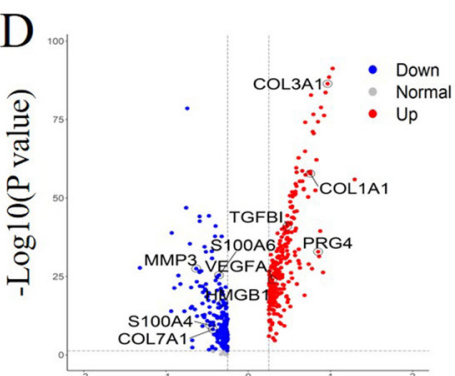

PreHTC-1 vs PreHTC-2

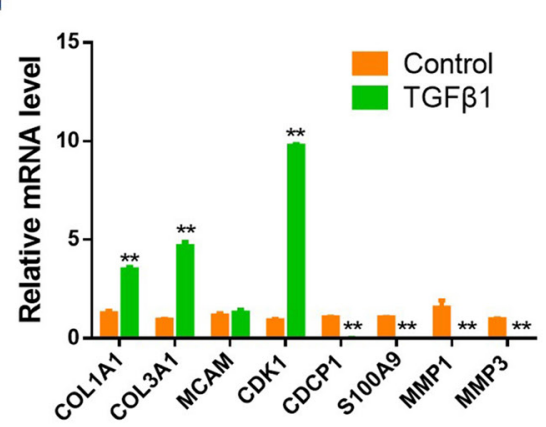

Figure 6 Activation of TGF $\beta$ signalling pathway attenuates the increase in CD318+ cells in degenerated meniscus. (A) The expression of TGF $\beta 1$ on merged and split t-distributed stochastic neighbourembedding map. (B) IHC staining of TGF $\beta 1$ on human healthy meniscus and degenerated meniscus. $n=6,{ }^{*} p<0.01$. (C) Volcano plot comparing the gene expression between FC-1 and FC-2. Each plot represents one gene. (D) Volcano plot comparing the gene expression between PreHTC-1 and PreHTC-2. Each plot represents one gene. (E) Human degenerated meniscus cells were treated with $5 \mathrm{ng} / \mathrm{mL}$ TGF $\beta 1$ for 48 hours or 96 hours. PBS was used as a negative control. CD318 expression was determined by flow cytometry ( $\mathrm{n} \geq 5$ ). * vs control, $\mathrm{p}<0.05 ; \&$ vs TGF $\beta 1$ (48hours), $\mathrm{p}<0.05$. (F) Human degenerated meniscus cells were treated with $5 \mathrm{ng} / \mathrm{mL}$ TGF $\beta 1$ or PBS as negative control. The expression of indicated marker genes were detected by qRT-PCR. $n=3,{ }^{* *} p<0.01$.

\section{DISCUSSION}

An increasing number of studies are supporting the idea that cell-based strategies effectively improve meniscus repair and regeneration. ${ }^{32}{ }^{33}$ However, it is still not clear which cell type is most effective for meniscus repair. ${ }^{3435}$ Recently, meniscus stem/ progenitor cells have been considered as the most suitable cell type for meniscus injury repair due to them having the same tissue origin and histocompatibility, ${ }^{3637}$ however, the characteristics, marker genes and isolation methods of human meniscus progenitor cells have not yet been fully elucidated. Gamer et al isolated meniscus progenitor cells from mice meniscus grown in explant cultures, and carried out flow cytometry analysis to show that these cells highly expressed CD44 and Sca-1. ${ }^{7}$ Shen et al digested human meniscus using collagenase and seeded the cells at a low density to form colonies. The subsequent flow cytometry analysis showed that these cell highly expressed CD90 (THY1) and CD105 (ENG), and the intra-articular injection of these cells promoted rat meniscus regeneration and ameliorated OA. ${ }^{37}$ Our scRNA-seq results also show the high expression of CD90 and CD105 in FCP, however, they were also highly expressed in FC-1 and FC-2. Thus, CD90 and CD105 were not markers specific to meniscus progenitor cells.

In our scRNA-seq results, EC was found to exist at the start of the pseudospace trajectory, which plays an important role in the development, degeneration and repair of the meniscus. ${ }^{38} 39$ Miller and Rydell isolated meniscus EC for the first time in 1993, and proved these cells had the ability to self-renew and maintain their characteristics after 10 passages. ${ }^{40} \mathrm{EC}$ is not only able to generate vessels to maintain blood supply, but also promote the migration of meniscus cells. Yuan et al found that EC could enhance meniscus cell migration by activating endothelin signalling. ${ }^{41}$ Notably, we identified CD146 specifically expressed in EC and FCP, suggesting that CD146 + cells can be used in cell-based scaffolding for meniscus injury repair, and may also be a target for recruitment of meniscus progenitor cells by growth factors to participate in meniscus injury repair in cell-free strategies.

We identified three cell clusters specific to degenerated meniscus, including MoDC, HTC and DegP, where DegP is a novel cluster and has the characteristics of progenitor cells. Our scRNA-seq demonstrated that the expression of DegP markers, such as GAS1, RAB3B and CD318, increased rapidly at the end of the differentiation of FCP to DegP, which was contrary to the normal differentiation procedure, suggesting that this differentiation process was the result of an aberrant cellular state. IHC staining also showed that GAS1 and RAB3B were highly expressed in meniscus with severe lesions, which was accompanied by cell proliferation. CD318 has been previously demonstrated to be highly expressed in haematopoietic progenitors ${ }^{42}$ 
and muscle progenitors. ${ }^{43}$ Iwata et al revealed that CD318 is a CD146 negative subset of bone marrow fibroblasts and regulates cytokine expression. ${ }^{44}$ Previous studies have shown that inflammatory cytokines such as IL-1 $\beta$ and TNF- $\alpha$ induce meniscus metabolic responses and result in degeneration. ${ }^{2845}$ In our study, IL-1 $\beta$ was used to induce the inflammatory response in human meniscus cells. We demonstrated that IL-1 $\beta$ decreased CD146+ cells and increased CD318 + cells in both healthy and degenerated meniscus cells. These results demonstrate that DegP plays a crucial role in meniscus degeneration and may be used as a marker to evaluate meniscus degeneration or as a target for the treatment of meniscus degeneration.

TGF $\beta$ is widely used in meniscus tissue engineering, owing to its promotion of meniscus injury repair and regeneration through the promotion of fibrochondrocyte proliferation and recruitment of meniscus progenitor cells. ${ }^{304647}$ TGF $\beta$ also regulates the meniscus degeneration process, while the postnatal deletion of TGF $\beta$ signalling reporter ALK5 accelerates meniscus degeneration. ${ }^{31}$ Our scRNA-seq results showed that TGF $\beta 1$ is highly expressed in FC-1 and FC-2, and that its overall expression in degenerated meniscus is decreased. Treatment with TGF $\beta 1$ has been previously found to enhance the mechanical properties of tissue-engineered fibrocartilage. ${ }^{48}$ Our results revealed that TGF $\beta 1$ attenuated the proportion of CD318+ cells in human degenerated meniscus, suggesting that TGF $\beta 1$ may be used to suppress meniscus degeneration.

In conclusion, our scRNA-seq results provided a clearer and more consistent definition of the cellular components of human meniscus, and the ways in which specific clusters contribute to meniscus development and aberrant degeneration. Our analysis identified the meniscus progenitors with potential in meniscus tissue engineering. We also demonstrated an important mechanism of meniscus degeneration and provided experimental evidence for a therapeutic strategy.

Acknowledgements We thank Yujie Liu and Bo Zhang from NovelBioinformatics for the support of bioinformatics analysis.

Contributors All listed authors meet the criteria for authorship and have contributed to the study design, data generation, data analysis, manuscript writing and manuscript review.

Funding This study was funded by the National Natural Science Foundation of China (no. 81874016, 81672145, 81472101, 81572119) and the Science and Technology Project of Guangzhou City, China (201710010164).

\section{Competing interests None declared.}

Patient consent for publication Not required.

Ethics approval Protocol approved by the Ethical Committee of The First Affiliated Hospital of Sun Yat-sen University.

Provenance and peer review Not commissioned; externally peer reviewed.

Data availability statement Data are available in a public, open access repository. The single-cell RNA-seq data, quality control information and cluster information are available at the NCBI's Gene Expression Omnibus (GEO) data repository with the accession ID GSE133449.

Open access This is an open access article distributed in accordance with the Creative Commons Attribution 4.0 Unported (CC BY 4.0) license, which permits others to copy, redistribute, remix, transform and build upon this work for any purpose, provided the original work is properly cited, a link to the licence is given, and indication of whether changes were made. See: https://creativecommons.org/ licenses/by/4.0/.

ORCID iDs

Hao Sun https://orcid.org/0000-0001-8829-0468

Zhiqi Zhang https://orcid.org/0000-0001-9086-2453

\section{REFERENCES}

1 Makris EA, Hadidi P, Athanasiou KA. The knee meniscus: Structure-function, pathophysiology, current repair techniques, and prospects for regeneration. Biomaterials 2011;32:7411-31.
2 Clark CR, Ogden JA. Development of the menisci of the human knee joint. Morphological changes and their potential role in childhood meniscal injury. J Bone Joint Surg Am 1983;65:538-47.

3 Danso EK, Oinas JMT, Saarakkala S, et al. Structure-Function relationships of human meniscus. J Mech Behav Biomed Mater 2017;67:51-60.

4 Cheung HS. Distribution of type I, II, III and V in the pepsin solubilized collagens in bovine menisci. Connect Tissue Res 1987;16:343-56.

5 Huang $\mathrm{H}$, Wang S, Gui J, et al. A study to identify and characterize the stem/progenitor cell in rabbit meniscus. Cytotechnology 2016;68:2083-103.

6 Seol D, Zhou C, Brouillette MJ, et al. Characteristics of meniscus progenitor cells migrated from injured meniscus. J Orthop Res 2017;35:1966-72.

7 Gamer LW, Shi RR, Gendelman A, et al. Identification and characterization of adult mouse meniscus stem/progenitor cells. Connect Tissue Res 2017;58:238-45.

8 Crema MD, Guermazi A, Li L, et al. The association of prevalent medial meniscal pathology with cartilage loss in the medial tibiofemoral compartment over a 2-year period. Osteoarthritis Cartilage 2010;18:336-43.

9 Sihvonen R, Paavola M, Malmivaara A, et al. Arthroscopic partial meniscectomy versus placebo surgery for a degenerative meniscus tear: a 2-year follow-up of the randomised controlled trial. Ann Rheum Dis 2018;77:188-95.

10 Fuller ES, Smith MM, Little CB, et al. Zonal differences in meniscus matrix turnover and cytokine response. Osteoarthritis Cartilage 2012;20:49-59.

11 Fuller $E$, Little CB, Melrose J. Interleukin- $1 \alpha$ induces focal degradation of biglycan and tissue degeneration in an in-vitro ovine meniscal model. Exp Mol Pathol 2016;101:214-20.

12 Herwig J, Egner E, Buddecke E. Chemical changes of human knee joint menisci in various stages of degeneration. Ann Rheum Dis 1984;43:635-40.

13 Svensson V, Natarajan KN, Ly L-H, et al. Power analysis of single-cell RNA-sequencing experiments. Nat Methods 2017;14:381-7.

14 Tian L, Dong X, Freytag S, et al. Benchmarking single cell RNA-sequencing analysis pipelines using mixture control experiments. Nat Methods 2019;16:479-87.

15 Baryawno N, Przybylski D, Kowalczyk MS, et al. A cellular taxonomy of the bone marrow stroma in homeostasis and leukemia. Cell 2019;177:1915-32.

$16 \mathrm{Ji}$ Q, Zheng Y, Zhang G, et al. Single-cell RNA-seq analysis reveals the progression of human osteoarthritis. Ann Rheum Dis 2019;78:100-10.

17 Dunn SL, Soul J, Anand S, et al. Gene expression changes in damaged osteoarthritic cartilage identify a signature of non-chondrogenic and mechanical responses. Osteoarthritis Cartilage 2016:24:1431-40.

18 Gunja NJ, Athanasiou KA. Passage and reversal effects on gene expression of bovine meniscal fibrochondrocytes. Arthritis Res Ther 2007;9.

19 Baker J, Falconer AMD, Wilkinson DJ, et al. Protein kinase D3 modulates MMP1 and MMP13 expression in human chondrocytes. PLoS One 2018;13:e0195864.

20 Won Y, Shin Y, Chun C-H, et al. Pleiotropic roles of metallothioneins as regulators of chondrocyte apoptosis and catabolic and anabolic pathways during osteoarthritis pathogenesis. Ann Rheum Dis 2016;75:2045-52.

21 Puram SV, Tirosh I, Parikh AS, et al. Single-cell transcriptomic analysis of primary and metastatic tumor ecosystems in head and neck cancer. Cell 2017; 171:1611-24.

22 Berent-Maoz B, Montecino-Rodriguez E, Signer RAJ, et al. Fibroblast growth factor-7 partially reverses murine thymocyte progenitor aging by repression of INK4A. Blood 2012:119:5715-21.

23 Loh C, Park S-H, Lee A, et al. Tnf-Induced inflammatory genes escape repression in fibroblast-like synoviocytes: transcriptomic and epigenomic analysis. Ann Rheum Dis 2019;78:1205-14.

24 McGovern N, Schlitzer A, Gunawan M, et al. Human dermal CD14+ cells are a transient population of monocyte-derived macrophages. Immunity 2014;41:465-77.

25 Alaaeddine N, Antoniou J, Moussa M, et al. The chemokine CCL20 induces proinflammatory and matrix degradative responses in cartilage. Inflamm Res 2015:64:721-31.

26 Chen Y-J, Chang W-A, Wu L-Y, et al. Systematic analysis of transcriptomic profile of chondrocytes in osteoarthritic knee using next-generation sequencing and bioinformatics. J Clin Med 2018;7:E535.

27 Worthley DL, Churchill M, Compton JT, et al. Gremlin 1 identifies a skeletal stem cell with bone, cartilage, and reticular stromal potential. Cell 2015;160:269-84.

$28 \operatorname{Cook}$ AE, Cook JL, Stoker AM. Metabolic responses of meniscus to IL-1 $\beta$. J Knee Surg 2018:31:834-40.

29 McNulty AL, Rothfusz NE, Leddy HA, et al. Synovial fluid concentrations and relative potency of interleukin-1 alpha and beta in cartilage and meniscus degradation. J Orthop Res 2013;31:1039-45.

30 Muhammad $\mathrm{H}$, Schminke B, Bode C, et al. Human migratory meniscus progenitor cells are controlled via the TGF- $\beta$ pathway. Stem Cell Reports 2014;3:789-803.

31 Wang Q, Tan Q, Xu W, et al. Postnatal deletion of $A / k 5$ gene in meniscal cartilage accelerates age-dependent meniscal degeneration in mice. J Cell Physiol 2019;234:595-605.

32 Shimomura K, Rothrauff BB, Hart DA, et al. Enhanced repair of meniscal hoop structure injuries using an aligned electrospun nanofibrous scaffold combined with a mesenchymal stem cell-derived tissue engineered construct. Biomaterials 2019;192:346-54 
33 Zhang Z-Z, Zhou Y-F, Li W-P, et al. Local administration of magnesium promotes meniscal healing through homing of endogenous stem cells: a proof-of-concept study. Am J Sports Med 2019;47:954-67.

34 Moradi L, Vasei M, Dehghan MM, et al. Regeneration of meniscus tissue using adipose mesenchymal stem cells-chondrocytes co-culture on a hybrid scaffold: In vivo study. Biomaterials 2017;126:18-30.

35 Zellner J, Pattappa G, Koch M, et al. Autologous mesenchymal stem cells or meniscal cells: what is the best cell source for regenerative meniscus treatment in an early osteoarthritis situation? Stem Cell Res Ther 2017;8.

36 Gui J, Zhang J, Huang H. Isolation and characterization of meniscus derived stem cells from rabbit as a possible treatment for damaged meniscus. Curr Stem Cell Res Ther 2015;10:353-63.

37 Shen W, Chen J, Zhu T, et al. Intra-Articular injection of human meniscus stem/progenitor cells promotes meniscus regeneration and ameliorates osteoarthritis through stromal cell-derived factor-1/CXCR4-mediated homing. Stem Cells Trans/ Med 2014;3:387-94.

38 Ashraf S, Wibberley H, Mapp PI, et al. Increased vascular penetration and nerve growth in the meniscus: a potential source of pain in osteoarthritis. Ann Rheum Dis 2011;70:523-9.

39 Rai MF, Patra D, Sandell LJ, et al. Transcriptome analysis of injured human meniscus reveals a distinct phenotype of meniscus degeneration with aging. Arthritis Rheum 2013;65:2090-101.

40 Miller RR, Rydell PA. Primary culture of microvascular endothelial cells from canine meniscus. J Orthop Res 1993;11:907-11.
41 Yuan X, Eng GM, Arkonac DE, et al. Endothelial cells enhance the migration of bovine meniscus cells. Arthritis Rheumatol 2015;67:182-92.

42 Takeda H, Fujimori Y, Kai S, et al. CD318/CUB-domain-containing protein 1 expression on cord blood hematopoietic progenitors. Exp Ther Med 2010;1:497-501

43 Uezumi A, Nakatani M, Ikemoto-Uezumi M, et al. Cell-surface protein profiling identifies distinctive markers of progenitor cells in human skeletal muscle. Stem Cell Reports 2016:7:263-78.

44 Iwata M, Torok-Storb B, Wayner EA, et al. Cdcp1 identifies a CD146 negative subset of marrow fibroblasts involved with cytokine production. PLoS One 2014;9:e109304.

45 Behrendt $\mathrm{P}$, Häfelein K, Preusse-Prange A, et al. II-10 ameliorates TNF- $\alpha$ induced meniscus degeneration in mature meniscal tissue in vitro. BMC Musculoskelet Disord 2017;18:197.

46 Lee $\mathrm{CH}$, Rodeo SA, Fortier LA, et al. Protein-releasing polymeric scaffolds induce fibrochondrocytic differentiation of endogenous cells for knee meniscus regeneration in sheep. Sci Transl Med 2014:6:266ra171.

47 Zhang Z-Z, Chen Y-R, Wang S-J, et al. Orchestrated biomechanical, structural, and biochemical stimuli for engineering anisotropic meniscus. Sci Trans/ Med 2019;11:eaao0750.

48 MacBarb RF, Makris EA, Hu JC, et al. A chondroitinase-ABC and TGF- $\beta 1$ treatment regimen for enhancing the mechanical properties of tissue-engineered fibrocartilage. Acta Biomater 2013;9:4626-34. 